\title{
ORTHODONTIC-RELATED POSTS ON SOCIAL MEDIA DURING COVID-19 EPIDEMIC
}

\author{
Reem A. Alansari*
}

\begin{abstract}
Objectives: To better understand people's orthodontic-related experiences, attitudes, and behaviors during the COVID-19 epidemic through their orthodontic-related social media posts.

Materials and Methods: This was a cross-sectional study. Multiple social media platforms were searched for orthodontic-related posts during March and April of 2020. Patterns were analyzed, coded, and categorized using qualitative thematic analysis.

Results: Social media posts revealed multiple themes that revolved around orthodontic treatment side effects, existing and future treatment, negative and positive perceptions of providers, and worrying about multiple aspects of the COVID-19 epidemic.

Conclusion: Patients generally prioritize personal and public health and accept orthodontic treatment delays provided they are reassured, and properly supported and educated. Social media platforms may provide an effective method for patients to voice their experiences and reach out to their practitioners during times of pandemics and they may be effective methods for practitioners to reach out to their existing patients and prospective patients.
\end{abstract}

KEY WORDS: Orthodontics, braces, qualitative, aligners, experience.

\section{INTRODUCTION}

During the past decade, social media use has increased progressively among the world's inhabitants. Facebook, Twitter, Instagram, Snapchat and other social media platforms have become parts of our daily lives, with billions of posts people used to share thoughts, photographs, opinions and to connect socially. People also use these platforms to share some aspects of their health and request advice from providers and other social media users, including posts and questions related to dental health. For example, a study found that 1 in 8 social media users expressed effects of dental pain or asked for advice on how to manage it on Twitter ${ }^{1}$. In another study, one in seven people reported sending posts about their braces, and $25 \%$ reacted to posts about braces from their followed social media accounts ${ }^{2}$.

\footnotetext{
* Assistant Professor, Department of Orthodontics, Faculty of Dentistry, King Abdulaziz University, Jeddah, Saudi Arabia
} 
These online platforms may provide a genuine and authentic way to get to people's feelings and experiences since they may feel unobserved and less restricted online in comparison to real life ${ }^{3,4}$. This provides a database of authentic data about patient experiences through orthodontic treatments, representing a useful resource to gain knowledge about people's perceptions of orthodontic treatments ${ }^{5}$.

Coronavirus Disease-2019 (COVID-19), a primarily respiratory infection caused by the Severe Acute Respiratory Syndrome Virus-2 (SARSCoV-2), has swept the world in a pandemic, infecting nearly 3 million cases worldwide and forcing governments and populations to take strong measures of social distancing ${ }^{6}$. Saudi Arabia has been involved in this pandemic with more than 18,000 cases confirmed by the end of April, 2020 ${ }^{6}$. COVID-19 is creating unique circumstances and challenges affecting patients' orthodontic-related experiences. As a result of the curfew policies enforced in Saudi Arabia, people have been mostly confined at home and orthodontics clinics have been closed for fear of spreading the virus among patients and healthcare professionals. Patients undergoing orthodontic treatment may have been experiencing higher levels of psychological distress during the pandemic than their usual level of anxiety ${ }^{7}$. Social distancing and curfew policies have led to an increase in utilization of social media platforms ${ }^{8}$. Orthodontic patients, therefore, may well be posting their experiences related to their treatment. These experiences may represent an important glimpse on the experiences, attitude and behaviors of orthodontic patients during the exceptional situation of pandemics.

The aim of this study is to better understand orthodontic people's subjective perceptions and experiences in relation to orthodontic treatments during the COVID-19 pandemic by analyzing their social media posts qualitatively.

\section{MATERIALS AND METHODS}

This was a cross-sectional study. The author collected social media posts related to orthodontic treatment posted by lay people, non-providers, on Twitter and Facebook during the months of March and April 2020 (coinciding with the establishment of the COVID-19 pandemic). Instagram was not used because it only allows for searching its platform using hashtags and hashtags are rarely used by patients in their posts. The following keywords were used to search Twitter and Facebook posts and hashtags both in their English form and in their Arabic translations: "orthodontist", "orthodontic", "braces", "aligners" within posts from Saudi Arabia in all platforms. The search was constricted using the apps to those originating from Saudi Arabia during March and April 2020. Posts and replies by orthodontic patients were included while those by providers were excluded.

Upon identification of a post that met the inclusion criteria, the post was entered into the data set. The username attached to the posts were anonymized with assigned identifiers. Posts by the same user were assigned the same identifier.

Simultaneous with data collection, qualitative thematic data analysis began by line-by-line assignment of codes that describe the meaning contained in each post ${ }^{9}$. Subsequently, categories with shared meanings were identified and themes with shared categories were created. Analysis was a reiterative process, following qualitative methodology. In other words, previously assigned codes and categories were adjusted according to the data that subsequently emerged in a process that progressively accumulates meaning and content of each category and theme. According to the "data saturation" principle, data collection continued until identification of new posts stopped leading to creation of new codes. 


\section{RESULTS}

Qualitative analysis of the data generated multiple themes that revolved around being in pain, existing treatment, future treatment, perception of providers, and worrying about multiple aspects of the COVID-19 epidemic. Themes are described below with supportive quotes.

\section{Reactions to the Decision to Suspend Clinic Activities}

Some patients described in their posts their acceptance of their providers' decisions to close clinics in accordance with local regulations. Some actually praised providers for sacrificing their income for the safety of patients and for the country. Patients also replied to the social media accounts of their providers expressing understanding and support for the decision and praying that the pandemic will resolve soon.

"This is better for everyone. That's the right thing to do"

\section{“...It's not easy to lose your livelihood”}

Another type of reaction was concern about whether clinics would refund portions of the paid treatment fees and some expressed displeasure with regards to the lack of clarity by some providers with regards to what would happen with the fees.

"...and what would happen with the thousands you already collected from us?"

Some patients have also interacted with each other in response to clinic closure, providing advice about what to do, support and clarifications. For example, a patient responded to a post of another patient asking what she would do with her braces by saying:

"Don't worry about your braces now! Even if an orthodontist was available, no one should be going to clinics now. We should all prioritize our health and the health of others".
Some patients were asking fellow patients about clinics that are still seeing patients because they still wanted their treatment to progress.

"Does anyone know an open clinic in...? I am almost done with my treatment and I want to finish"

A related category of quotes was an expression of relief and the advantage of the decision to be treated with clear aligners.

"The situation we are going through in this period proves that clear aligners are way better than other braces"

"Thank God I have trays!"

\section{Concerns of the Impact of Prolongation of the} Pandemic on the Orthodontic Treatment

Some orthodontic patients expressed worries about the possible negative consequences of the prolonged pandemic on their orthodontic treatment. They inquired what might happen to them if they are not seen by an orthodontist for a prolonged period. They worried about unpredictable changes.

"What will happen to my teeth if I don't get seen?!”

"When is the quarantine going to be over?"

Another category of quotes expressed being worried about the esthetic appearance and the hygienic status of their braces.

"My braces look and feel disgusting I cannot take it anymore"

Many patients expressed remarks of their disappointment in the delay of the end of their treatment and for having to stay in their braces more than they would have were it not for the pandemic. A participant rhetorically remarked in the context of a conversation about the delay of orthodontic treatment due to the closures resulting from the pandemic, saying:

"Orthodontic treatment is supposed to make you look better. Why am so unlucky that it is making me worse? When is this nightmare going to end?" 


\section{Pain and Side Effects of Treatment}

A myriad of posts by patients described difficulties they were going through in relation to their orthodontic treatment and the imposed curfew. Pain was one of the major concerns participants expressed and asked for advice from others about.

"it's slashing my cheek open and I can't go out. What do I do?"

Patients also posted experiences related to broken pieces of their braces, discomfort with their wires, and other sources of pain and discomfort.

Participants also described not receiving sufficient advice and follow up by their orthodontists in this regard. This is a remark of a patient who's expressing dissatisfaction with how little advice there are getting from orthodontists compared to other providers:

"What about us? Where are the orthodontists?"

\section{Direct Interaction with Providers}

Patients interacted directly with the accounts of their providers. Many asked technical questions about thing going wrong with their appliances or about treatment progression.

"Doctor, the band is out if its place, what's the solution"

"I have reached my last tray, what do I do?"

Patients expressed praise and prayers for the orthodontists posting educational material, photographs, videos and articles about how to deal with braces and about orthodontics in general.

"Thank you, Dr. ... your posts are very useful, and I am lucky to be your patient"

"Your recommendations are very valuable, and I appreciate what you are doing to help us get through this"

\section{Initiation of orthodontic treatment}

Furthermore, a category of posts by prospective patients inquired about the merits of orthodontic treatment, the pros and cos, the costs and the side effects, expressing desire to start treatment despite the clinics being closed at the time.

"I can't wait for your clinic to re-open so I can start treatment!"

"Is orthodontic treatment painful?"

"Do braces make you look cuter?"

\section{DISCUSSION}

This study explored the subjective experiences posted by lay people on social media platforms in Saudi Arabia in relation to orthodontic treatment during the period when COVID-19 is sweeping the world in a pandemic. COVID-19 has caused a tremendous psychological impact on the general population as a whole and on patients with various maladies $^{13-11}$. Orthodontic treatment may be associated with anxiety among patients and parents in usual living situations and this anxiety may impact their quality of life ${ }^{14,15}$. It is important therefore to investigate and understand how orthodontic patients' experiences and anxieties may be shaped during the period of a pandemic. This study used a qualitative analysis approach of social media data and revealed important themes of thoughts, feelings, attitude, and behaviors that orthodontic patients expressed during the current pandemic in relation to orthodontic treatment and the uncertainty and restrictions on available orthodontic services. These themes are of interest to orthodontists and treatment providers in general. It is important for providers to communicate with their patients effectively during this period using remote communication methods as this was demonstrated to be of help in the past and during the current pandemic ${ }^{16,17}$. Learning about people's experiences, thoughts, and feelings may guide communication and educational efforts during these difficult times and may help providers and patients prepare and deal with future situations of similar nature as well.

Orthodontic clinics may have wondered about how their patients would feel about restrictions to services in the current context. The data have 
been reassuring to an extent about orthodontic patient attitudes. Patients have in fact expressed appreciation for the clinic closures and prioritized personal and public health. They seemed to be to be alert and to ask when side effects occurred. Nonetheless, patients expressed concerns about the impact of the pandemic on the previously paid cost of their orthodontic treatment. It is well known that, depending on the healthcare and insurance systems in a given country, the cost of the treatment is a source of concern and may deter patients from undergoing and complying with orthodontic treatment ${ }^{18,19}$. Therefore, this is an important aspect that needs to be clarified and communicated well to patients in order to lower their anxiety as to what will happen to their paid fees in these uncertain times with significant financial implications.

This study provides reassurance to orthodontists and orthodontic clinics that interest in orthodontic services continues to be expressed in social media during the pandemic and at these financially difficult times. Providers and clinic owners may need to factor that in their planning and decision-making.

Patients appreciated educational teaching through social media platforms through these difficult times and praised providers that did that. There is previous evidence that social media is effective in communication with orthodontic patients ${ }^{5,16}$. It is especially important during times of pandemics where social media may be the only and most effective way to communicate with patients who are home isolated ${ }^{8}$.

An interesting finding in this study is that clear aligners were described to provide a piece of mind for some patients. This may be due to the fact that they do not impose an esthetic burden and therefore the delay in the treatment is less burdensome than traditional braces. Another explanation may be that treatment with aligners can progress with the patient moving to the following aligner, as planned by the provider, without the need for the clinic activation visit as the case with traditional braces.
This study derives its validity from the rich description of patient posts and derives validity from the reiterative analytic process. In other words, concepts that are formulated at the end of the analysis derive their credibility from being referenced to data derived from earlier stages of the analysis in a reiterative manner. One of this study's limitations is it's focus on two social media platform because of the ease of data retrieval. Patients may share additional ideas on other social media platforms such as Snapchat (where posts do not last for more than 24 hours) and Instagram (where posts are not easily searchable without having hashtags). Furthermore, the results of this study may be different in later stages of a pandemic. Future research that draws data from all available social media platforms and pursues data longitudinally is required to further elucidate patient experiences during pandemics and how role of social media in communication with patients during these difficult times. Another limitation may arise from the inability to generalize these orthodontic-related experiences because data were collected from people who live in Saudi Arabia and also from people chose to share their experiences on social media. Future research should be directed at widening the search to include other nations and to include other types of data such as phone interview or face-to-face video interviews.

\section{CONCLUSION}

- Patients generally prioritize personal and public health and accept orthodontic treatment delays provided they are reassured about the security of the costs they have already payed and as long as they are given enough information to get through problems which may arise.

- Social media platforms may provide an effective method for patients to voice their experiences and reach out for their practitioners during times of pandemics and they may be effective methods for practitioners to reach out to their existing patients and prospective patients. 
- Being treated by clear aligners may provide a less stressful and more effective treatment experience for patients during times of pandemics.

- Further studies are needed during the times of pandemics to explore these themes quantitatively and guide orthodontic clinics in the best ways to communicate with their patients during these difficult times.

\section{REFERENCES}

1. Heaivilin N, Gerbert B, Page JE, Gibbs JL. Public health surveillance of dental pain via Twitter. J Dent Res. 2011;90(9):1047-1051. doi:10.1177/0022034511415273

2. Henzell M, Knight A, Antoun JS, Farella M. Social media use by orthodontic patients. N Z Dent J. 2013;109(4):130133.

3. Maczewski M. Exploring Identities Through the Internet: Youth Experiences Online. Child \& Youth Care Forum. 2002;31(2):111-129. doi:10.1023/A:1015322602597

4. Barber SK, Lam Y, Hodge TM, Pavitt S. Is social media the way to empower patients to share their experiences of dental care? The Journal of the American Dental Association. 2018;149(6):451-459.e9. doi:10.1016/j.adaj.2018.01.007

5. Papadimitriou A, Kakali L, Pazera P, Doulis I, Kloukos D. Social media and orthodontic treatment from the patient's perspective: a systematic review. Eur J Orthod. doi:10.1093/ejo/cjz029

6. The WHO. The WHO COVID-19 Dashboard. April 2020. https://covid19.who.int/.

7. Differences of psychological status of TMD patients, orthodontic patients and the general population during the $\mathrm{CO}$ VID-19 epidemic: a cross-sectional study - Abstract - Europe PMC. https://europepmc.org/article/ppr/ppr129387. Accessed April 28, 2020.

8. Wiederhold BK. Social Media Use During Social Distancing. Cyberpsychol Behav Soc Netw. April 2020. doi:10.1089/cyber.2020.29181.bkw

9. Braun V, Clarke V. Using thematic analysis in psychology. Qualitative Research in Psychology. 2006;3(2):77-101. doi:10.1191/1478088706qp063oa
10. Liu D, Ren Y, Yan F, et al. Psychological Impact and Predisposing Factors of the Coronavirus Disease 2019 (COVID-19) Pandemic on General Public in China. Rochester, NY: Social Science Research Network; 2020. doi:10.2139/ ssrn. 3551415

11. Huang Y, Zhao N. Generalized anxiety disorder, depressive symptoms and sleep quality during COVID-19 epidemic in China: a web-based cross-sectional survey - Abstract - Europe PMC. Psychiatry Research. 2020;288(112954). https://europepmc.org/article/ppr/ppr122811. Accessed April 29, 2020.

12. Wang C, Pan R, Wan X, et al. Immediate Psychological Responses and Associated Factors during the Initial Stage of the 2019 Coronavirus Disease (COVID-19) Epidemic among the General Population in China. International Journal of Environmental Research and Public Health. 2020;17(5):1729. doi:10.3390/ijerph17051729

13. Sari Z, Uysal T, Karaman AI, Sargin N, Üre Ö. Does orthodontic treatment affect patients and parents anxiety levels? Eur J Orthod. 2005;27(2):155-159. doi:10.1093/ejo/ cjh072

14. Alsaadi T, Kassie S, El Hammasi K, et al. Potential factors impacting health-related quality of life among patients with epilepsy: Results from the United Arab Emirates. Seizure. 2017;53:13-17. doi:10.1016/j.seizure.2017.10.017

15. Bartlett BW, Firestone AR, Vig KWL, Beck FM, Marucha PT. The influence of a structured telephone call on orthodontic pain and anxiety. American Journal of Orthodontics and Dentofacial Orthopedics. 2005;128(4):435-441. doi:10.1016/j.ajodo.2004.06.033

16. Zhou X, Snoswell CL, Harding LE, et al. The Role of Telehealth in Reducing the Mental Health Burden from COVID-19. Telemedicine and e-Health. 2020;26(4):377-379. doi:10.1089/tmj.2020.0068

17. Gosney MBE. An Investigation into Factors Which May Deter Patients from Undergoing Orthodontic Treatment. British Journal of Orthodontics. 1985;12(3):133-138. doi:10.1179/bjo.12.3.133

18. Uribe F, Padala S, Allareddy V, Nanda R. Patients', parents', and orthodontists' perceptions of the need for and costs of additional procedures to reduce treatment time. American Journal of Orthodontics and Dentofacial Orthopedics. 2014; 145 (4, Supplement): S65-S73.doi:10.1016/j. ajodo.2013.12.015 